\title{
Measurement of the Shielding Effectiveness of Conducting Enclosures with the Image Theory
}

\author{
U. Paoletti ${ }^{1,2, *}$, H. Garbe ${ }^{3}$, and W. John ${ }^{2}$ \\ ${ }^{1}$ Faculty of Computer Science, Electrical Engineering and Mathematics, University of Paderborn, Warburger Straße 100, \\ 33098 Paderborn, Germany \\ ${ }^{2}$ Fraunhofer Institute Reliability and Microintegration, Department ASE, Gustav-Meyer-Allee 25, 13355 Berlin, Germany \\ ${ }^{3}$ Institute for Electrical Engineering and Measurement Science, University of Hanover, Appelstraße 9A, 30167 Hanover, \\ Germany \\ *Competence Network Future EMC/RF Modeling and Simulation Methodologies (FhG IZM / University of Paderborn / \\ University of Hanover), Germany
}

\begin{abstract}
Two simple techniques for the measurement of the shielding efficiency for the electric field of a symmetric enclosure are presented.y They allow measurements in the symmetry plane of the enclosure without disturbances $b$ the cables. Measurements in near and far field regions are possible. Comparisons with simulation results are shown. Both techniques are particularly suitable for the evaluation of numerical techniques. Limits of the concept of shielding efficiency of enclosures at high frequencies are also briefly discussed.
\end{abstract}

\section{Introduction}

The determination of the shielding efficiency $(S E)$ of an enclosure is important for the early estimation of the radiation emission from electronic equipment and their susceptibility to external electromagnetic interferences. Several publications confirm the importance of this topic (e.g. Li et al., 2000; Paul et al., 2003; Siah et al., 2003).

At frequencies higher than the resonance frequency of the enclosure, the shielding efficiency becomes strongly dependent on the spatial position inside the enclosure where it is calculated and, therefore, its definition loses part of its significance. Moreover, its calculation becomes more difficult and requires the use of more sophisticated numerical techniques, which take into account many details of the geometry such as apertures.

On the other hand, the correctness of the results calculated with numerical techniques in their developing stage or in innovative applications must be corroborated by experimental results. For a comparison of the results in a wide frequency range, a scalar quantity is preferable and the shielding efficiency is often chosen (Olyslager et al., 1999). However, to our knowledge it has been always measured on the internal

Correspondence to: U. Paoletti

(umberto.paoletti@izm.fraunhofer.de) surface of the enclosure by means of small monopoles introduced through the walls.

In the following, two simple measurement techniques are proposed, which allow the measurement of the shielding efficiency on a symmetry plane of the enclosure (Paoletti et al., $2004 b$,a). This can be useful for a direct even though invasive measurement at frequencies above the resonance frequency, as well as test for numerical techniques.

Comparisons with results obtained by a simulation technique based on a three dimensional extension (Paoletti et al., 2003) of the BiCG-FFT method (e.g. Sarkar et al., 1986; Catedra, 1995) will be shown.

Well conducting walls will be considered in the examples without the introduction of additional losses. This increases the quality factor of the enclosure, accentuates the spatial dependence of the shielding efficiency and can be therefore considered as an extreme situation, which is particularly difficult both in measuring and in simulating.

\section{Shielding Effectiveness}

\subsection{Definition and Limits}

The shielding effectiveness for the electric field $\left(S E_{E}\right)$ is defined as the inverse of the ratio expressed in $\mathrm{dB}$ between the norm of the electric field $\boldsymbol{E}_{t}$ at a point inside an enclosure and the norm of the field $\boldsymbol{E}_{i}$ at the same point without the enclosure:

$S E_{E}=20 \log _{10} \frac{\left\|\boldsymbol{E}_{i}\right\|}{\left\|\boldsymbol{E}_{t}\right\|}$

A similar definition exists for the magnetic field. In the following, however, only that for the electric field will be considered. The shielding effectiveness shown in Eq. (1) is dependent on the excitation. Usually an assumption of far field from the source is made and a linearly polarized plane wave is considered. Nevertheless, a dependence on the direction of incidence and on the polarization of the field remains. 


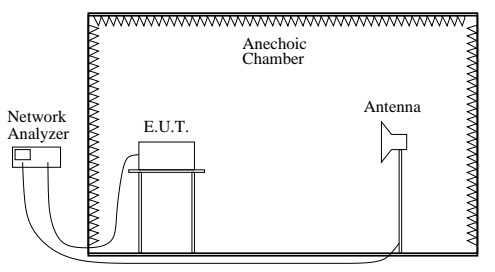

Fig. 1. Traditional measurement setup in the anechoic chamber.

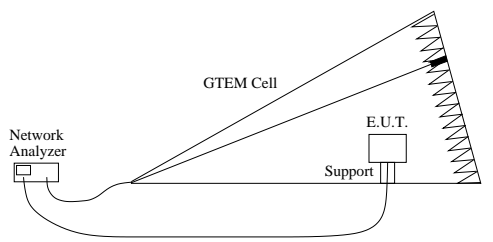

Fig. 2. Traditional measurement setup in the GTEM cell.

For frequencies above the resonance frequencies of the conducting cavity, superior modes can be excited and it is not possible to assume that the field inside the enclosure is constant along one direction.

The presence of apertures complicates the treatment, but an independence of the shielding effectiveness from one of the coordinate directions at high frequencies in general cannot be asserted. For these reasons, the shielding effectiveness alone is not sufficient for considering the effect of an enclosure to the electromagnetic radiation from electronic equipment at high frequencies. The interaction with the enclosure should be taken into account with numerical or pseudoanalytical techniques, which should consider also the position and orientation of the equipment in the enclosure. For the validation of such techniques, however, the shielding effectiveness is very well suitable.

\subsection{Traditional measurement setups}

A typical measurement setup is shown in Fig. 1. A coaxial cable is inserted through a wall of the enclosure (E.U.T). The center conductor acts as monopole antenna on the internal wall of the enclosure. The transmitting antenna and the enclosure are set in an anechoic chamber and connected to a spectrum analyzer or to a network analyzer.

Clearly this measurement setup allows the measurement of the shielding efficiency only on the walls of the enclosure. Moreover the presence of the cables may interfere with the measurement results.

Similar observations can be made for the measurement setup of Fig. 2, where the enclosure is set on a nonconducting support inside a GTEM cell.

\subsection{Equivalent use of spectrum or network analyzer}

For this type of measurement either a spectrum analyzer or a scalar network analyzer can be used. This can be easily verified with the equivalent circuit in Fig. 3, where antenna,

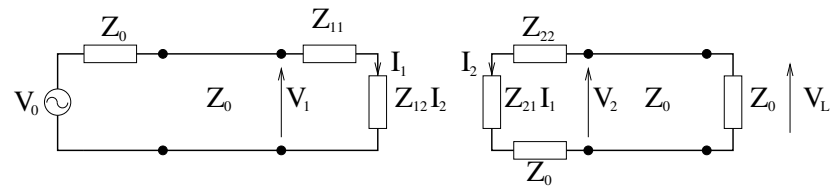

Fig. 3. Equivalent circuit of the measurement setup.

monopole and transmission medium have been substituted with the $Z$-representation of a two-port network. Generator impedance and input impedance of the measurement equipment are equal to the characteristic impedance $Z_{0}$ of the cables. The voltage at the port of the measurement equipment connected to the monopole has been indicated with $V_{L}$.

When a spectrum analyzer is used, the voltage $V_{L}$ on the load is measured. The norm of the electric field can be calculated by means of the antenna factor. In this case, however, it is not necessary to know the electric field, because the shielding effectiveness is a relative value and can be obtained directly from the voltages on the load $V_{L n}$ and $V_{L e}$ without and with the enclosure respectively:

$S E_{E}=20 \log _{10} \frac{\left|V_{L n}\right|}{\left|V_{L e}\right|}$

The expression of the voltage $V_{L}$ as function of the source voltage $V_{0}$ is:

$V_{L}=\frac{Z_{0} Z_{21}}{\left(Z_{0}+Z_{22}\right)\left(Z_{0}+Z_{11}\right)-Z_{12} Z_{21}} V_{0}$

The scattering matrix $\mathbf{S}$ can be derived from the impedance matrix $\mathbf{Z}$ of the two-port network with Eq. (4), where $\mathbf{I}$ is the identity matrix. The corresponding expression for the transmission parameter $S_{21}$ of the scattering matrix, which is measured with a network analyzer, is shown in Eq. (5).

$\mathbf{S}=\left(\mathbf{Z}+Z_{0} \mathbf{I}\right)^{-1}\left(\mathbf{Z}-Z_{0} \mathbf{I}\right)$

$S_{21}=\frac{2 Z_{0} Z_{21}}{\left(Z_{0}+Z_{22}\right)\left(Z_{0}+Z_{11}\right)-Z_{12} Z_{21}}$

It is therefore evident from Eqs. (3) and (5) that the shielding effectiveness can be equivalently measured with a scalar network analyzer by means of Eq. (6), where $S_{21 n}$ and $S_{21 e}$ are the transmission parameters measured without and with the enclosure respectively.

$S E_{E}=20 \log _{10} \frac{\left|S_{21 n}\right|}{\left|S_{21 e}\right|}$

\section{Measurement with the image theory}

\subsection{Measurement in the anechoic chamber}

For symmetric enclosures it is possible to make use of the image theory for measuring the shielding effectiveness. A first measurement setup makes use of a conducting surface 


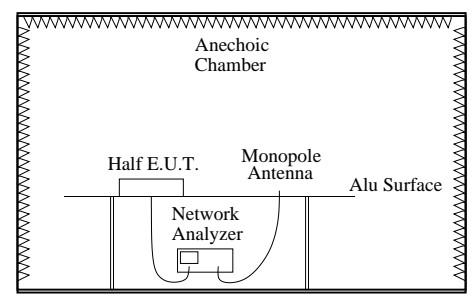

Fig. 4. Measurement setup with image theory in the anechoic chamber.

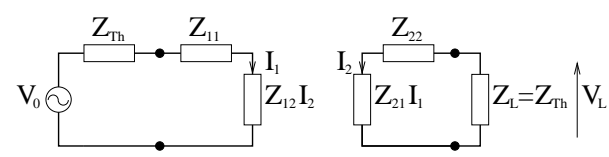

Fig. 5. Equivalent circuit for transmitting and receiving antennas in the simulations.

in an anechoic chamber (Paoletti et al., 2004b). A half enclosure is laid on an aluminum surface and connected to it by means of copper tape. Monopoles are set on the conducting surface inside and outside the enclosure, and fed with coaxial cables under the surface, as shown in Fig. 1 . The complete measurement setup is placed in an anechoic chamber.

By neglecting the finiteness of the conducting surface, the image theory can be applied. In this way, the half enclosure becomes a full one, and the monopoles become dipoles. Moreover, the cables disappear from the setup, and the dipoles in the equivalent configuration can be considered as fluctuating in the air. In order to neglect the finiteness of the conducting plane, its extension must be sensibly large in comparison with the dimension of the enclosure. However, as the $S E_{E}$ is calculated as the ratio between two measurements, both in the presence of the plane, its dimension does not need to be very large.

The symmetry of the complete enclosure and of the excitation ensures that the tangential electric field on the symmetry plane is zero for all frequencies. This justifies the application of the image theory and the equivalence of the two configurations.

\subsection{Equivalent circuits for simulations and measurements}

The simulations have been executed with two dipoles and a complete enclosure. Moreover the $S E_{E}$ has been calculated directly at the ports of the antennas, without introducing transmission lines. It is therefore important to verify, that the measurement and simulation results refer to the same quantities.

In the simulations the dipole inside the enclosure is fed by means of an equivalent Thevenin generator and the external dipole is closed on a load impedance $Z_{L}=Z_{T h}$. The reciprocity theorem ensures, that these results are the same as those for a source outside the enclosure with a passive antenna inside. The equivalent circuit is shown in Fig. 5.

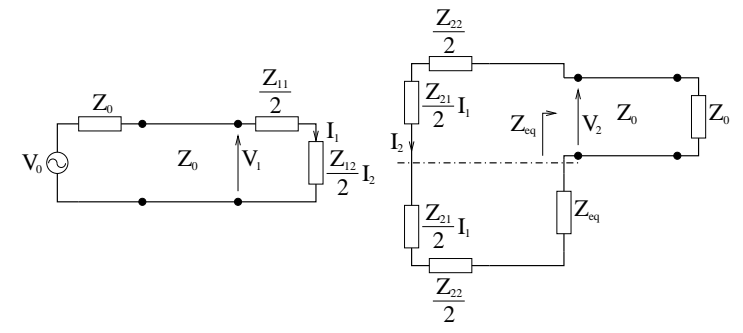

Fig. 6. Equivalent circuit for the measurements with the symmetry plane.

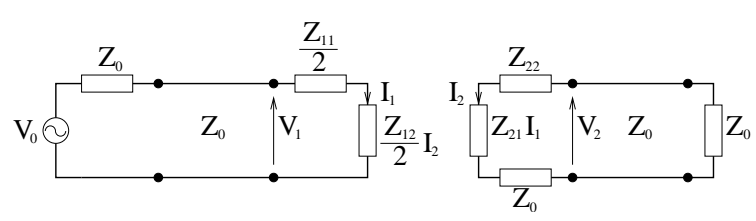

Fig. 7. Simplified equivalent circuit for the measurements.

The simulations are executed for several frequencies with and without the enclosure. The shielding efficiency is calculated from the two results for the voltage on the dipole outside the enclosure, whose dependency on the excitation $V_{0}$ is as follows:

$V_{L}=\frac{Z_{T h} Z_{21}}{\left(Z_{T h}+Z_{22}\right)\left(Z_{T h}+Z_{11}\right)-Z_{12} Z_{21}} V_{0}$

The circuit representation for the measurement results is shown in Fig. 6, where the parameters $Z_{11}, Z_{12}, Z_{21}$ and $Z_{22}$ refer to complete dipole antennas, as in the configuration for the simulations. After a rearrangement of the circuit elements, the equivalent circuit of Fig. 7 is obtained.

The transmission parameter $S_{21}$ can be calculated as follows:

$S_{21}=\frac{4 Z_{0} Z_{21}}{\left(2 Z_{0}+Z_{22}\right)\left(2 Z_{0}+Z_{11}\right)-Z_{12} Z_{21}}$

It is evident that the expressions for the shielding effectiveness for the simulations and the measurements calculated in Eq. (9) are the same, if generator and load impedances of the antennas in the simulations are set to $2 Z_{0}$ :

$S E_{E}=20 \log _{10} \frac{\left|V_{L n}\right|}{\left|V_{L e}\right|}=20 \log _{10} \frac{\left|S_{21 n}\right|}{\left|S_{21 e}\right|}$

where the subscripts $e$ and $n$ indicate with and without the enclosure respectively. The only artifice for a comparison of the results is, therefore, the use of generator and load impedances in the simulations, whose value is twice as that of the internal impedance of the network analyzer, that is:

$Z_{T h}=Z_{L}=2 Z_{0}$ 


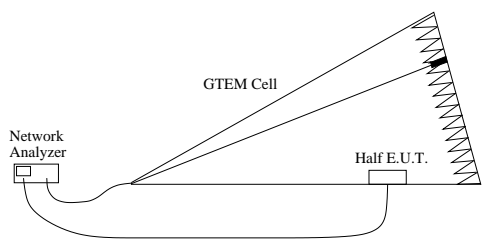

Fig. 8. Measurement setup with image theory in the GTEM cell.

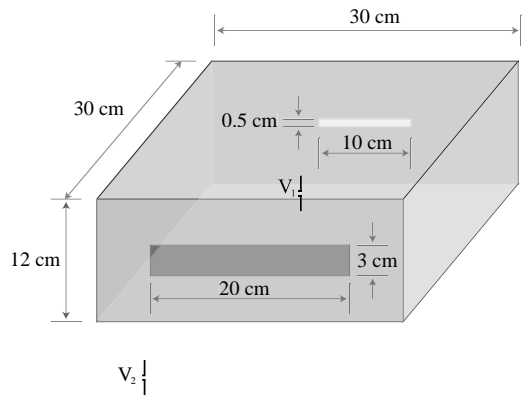

Fig. 9. Geometry of the enclosure.

\subsection{Measurement in GTEM cell}

The same principle can be applied for measurements in the GTEM cell (Paoletti et al., 2004a). In this case the half enclosure is laid on the floor of the cell and a monopole is introduced through the floor inside the enclosure. The effect of the cable can be once more neglected. A sketch of the measurement setup is shown in Fig. 8.

In this configuration only a monopole is necessary, in fact the second port of the network analyzer is connected to the port of the GTEM cell. This is an advantage in the lower frequency range, where the dipole is very short in comparison with the wavelength, and thus not very sensitive. A second important difference is that the incident field in the position of the enclosure assumes a distribution very similar to that of a linearly polarized plane wave. In this way the far field shielding effectiveness can be measured.

\section{Results}

An enclosure of dimensions $30 \times 30 \times 12 \mathrm{~cm}$ with a $20 \times 3 \mathrm{~cm}$ slot on the front wall and a $10 \times 0.5 \mathrm{~cm}$ slot on the back wall has been considered. This configuration, shown in Fig. 9, is the same used in Georgakopoulos et al. (2001), and results for the shielding efficiency are therefore available up to $1.5 \mathrm{GHz}$.

However, those measurement results have been obtained with the measurement setup of Fig. 1, with the monopole on an internal wall. The FDTD method has been used for the simulations.

For the present article we have used the measurement setups of Figs. 4 and 8. At first, a half enclosure of copper with $1.5 \mathrm{~mm}$ thick walls has been laid on a $2500 \times 1250 \times 1.5 \mathrm{~mm}$ aluminum surface, and connected to it by means of a $0.038 \mathrm{~mm}$ thick and $25.4 \mathrm{~mm}$ wide copper tape. A picture

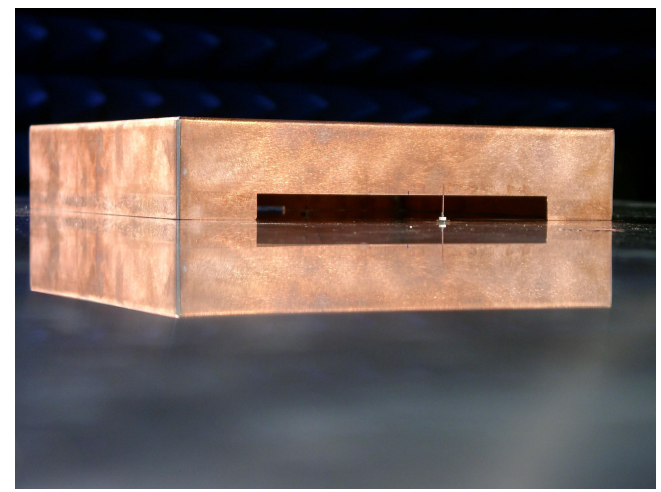

Fig. 10. Half enclosure on the aluminum surface in the anechoic chamber.

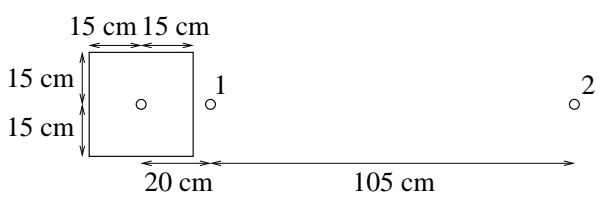

Fig. 11. Position of the monopole antennas.

of the half enclosure on the aluminum surface without copper tape is shown in Fig. 10.

The source and receiving monopoles were $2 \mathrm{~cm}$ long. The source monopole has been placed in the middle of the enclosure. Two measurements have been conducted with the receiving monopole placed outside of the enclosure respectively at $5 \mathrm{~cm}$ and $1.1 \mathrm{~m}$ distance from the front wall, as shown in Fig. 11.

Comparisons up to $2.5 \mathrm{GHz}$ of the measurement results with the simulation results obtained with the BiCG-FFT method (Paoletti et al., 2003) are shown in Figs. 12 and 13 respectively. In the simulations, $30 \times 30 \times 48$ segments have been used for the edges of the enclosure, and four segments have been used for each dipole.

The agreement with the receiving dipole at $5 \mathrm{~cm}$ from the front wall is very good. In the lower frequency range the signal captured by the short monopole was too weak for the sensitivity of the instrument and for the precision of the solution proposed for the calculation of the coupling between monopoles and enclosure.

The agreement with the receiving dipole at $1.1 \mathrm{~m}$ is also good, but the effect of the weakness of the signal is more noticeable, particularly for frequencies below $500 \mathrm{MHz}$. At these frequencies the shielding effectiveness for the electric field is still high, because no resonance of the enclosure appears.

For frequencies up to $2.5 \mathrm{GHz}$ the assumption of constant electric field along the shortest direction inside the enclosure is still valid for this enclosure, if the effect of the slots can be neglected and only the modes of a rectangular resonator are considered. In general, however, the apertures modify the field distribution inside the enclosure and irregularities 


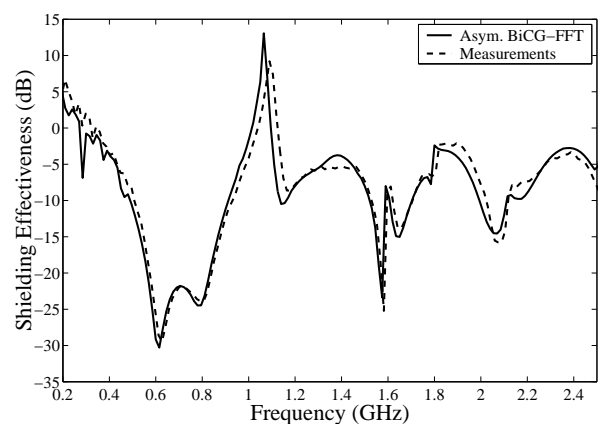

Fig. 12. Measurement and simulation results at $5 \mathrm{~cm}$.

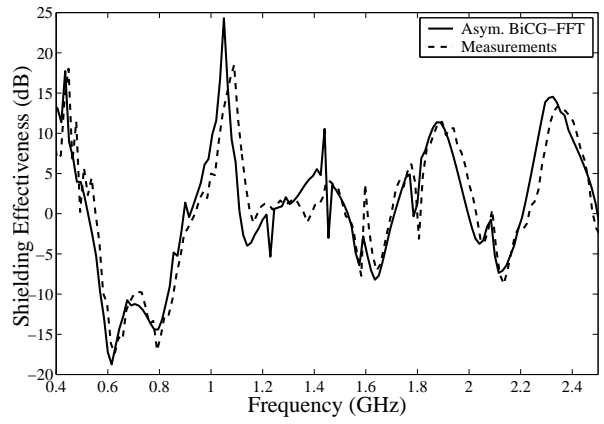

Fig. 13. Measurement and simulation results at $110 \mathrm{~cm}$.

are likely to appear at lower frequencies, depending on the aperture configuration.

With this measurement technique it is possible to conduct measurements in the whole symmetry plane. Therefore, it is possible to measure the effect of the apertures in the near field region without worrying about possible undesired coupling between cables and enclosure. It is also interesting to consider the possibility of using this technique for measuring the shielding effectiveness for sources in the far field region. To this purpose, a comparison with measurements in the GTEM cell has been made.

The same half enclosure has been laid on the floor of a GTEM 1250 according to the setup of Fig. 8. The monopole was $2.5 \mathrm{~mm}$ long and had a radius of $1 \mathrm{~mm}$. A picture of the setup is shown in Fig. 14.

Fig. 15 shows a comparison between the shielding effectiveness in the center of the enclosure calculated with an incident plane wave and with both measurement setups. For the measurement in the anechoic chamber, the monopole was at $1.1 \mathrm{~m}$.

All the curves agree up to $2.5 \mathrm{GHz}$. This confirms the correctness of the measurement setup in the GTEM cell. Moreover, it can be observed that the chosen distance for the observation point in the anechoich chamber is sufficient for having a good estimation of the shielding efficiency at much larger distance, apart from few frequencies where the presence of the dipoles generates isolated peaks. The peak near $1.4 \mathrm{GHz}$ due to the antenna inside the enclosure has been already observed in Georgakopoulos et al. (2001).

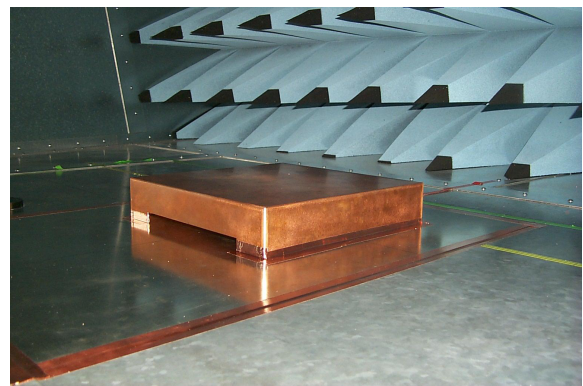

Fig. 14. Half enclosure in the GTEM cell.

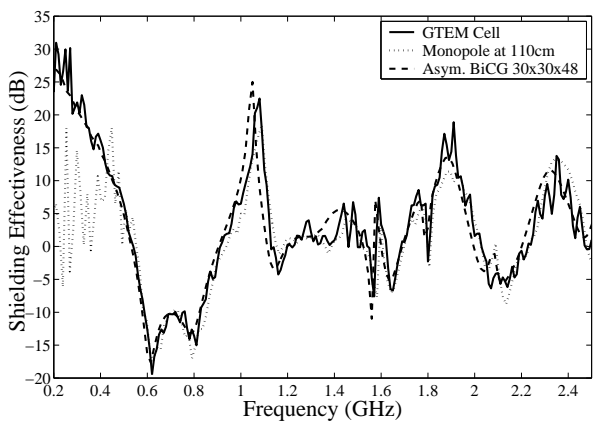

Fig. 15. Measurement and simulation results.

The measurement results of both techniques are in agreement up to the highest measured frequency, which is $3 \mathrm{GHz}$. However, the results above $2.5 \mathrm{GHz}$ have not been shown, because no agreement with simulation results has been obtained. At these frequencies, a more complex model for the monopole must be used.

From Fig. 15 it is also evident that the mentioned numerical error in the simulations below $500 \mathrm{MHz}$ is due to a not enough accurate model of the coupling between the far dipole and the enclosure, because it does not appear in the simulations with the plane wave.

Some artifacts are present in the measurement results with the GTEM cell under the form of an almost periodic oscillation in frequency domain. This corresponds to a standing wave inside the cell. From the period of oscillation the position of the reflection can be approximately obtained. The reflection seems to happen beyond the position of the enclosure and could be due to a bad matching of the cell. Further investigations have not been executed.

\section{Conclusions}

A few limits of the concept of shielding effectiveness for characterizing enclosures at high frequencies have been shown. Also some limits in the traditional measurement techniques have been outlined. Two measurement techniques based on the image theory have been proposed for symmetric enclosures. 
A first advantage is that they allow the measurement of the shielding efficiency in the whole symmetry plane. Secondly they allow to neglect the effect of the feeding cables. Measurement in near and far field are possible.

The model for the simulations is validated by means of equivalent circuits. Comparisons with simulation results show a very good agreement, particularly for measurements near the enclosure. In the lower frequency range the sensitivity of the receiving devices were too low, and the agreement below the first resonance frequency of the considered enclosure was poor.

Even though the utilization of these techniques for measuring the shielding effectiveness of existing enclosures is in some cases imaginable, the limitations imposed by the assumed symmetry of the whole configuration make these techniques more suitable for experimentally evaluating numerical techniques.

Acknowledgements. The reported $\mathrm{R}+\mathrm{D}$ work in this paper was carried out in the frame of the Eureka project MEDEAplus MESDIE A 509-Microelectronic EMC Design for High Density Interconnect and High Frequency Environment. The MESDIE project deals with the analysis and optimization of electromagnetic compatibility aspects on high speed and high density silicon and package design applications respectively.

In this frame the reported results are part of a task which aims at the modeling of electromagnetic radiation from IC and system level. The presented measuring techniques are intended for testing a calculation method for the effect of perforated conducting enclosures on the radiation. This particular research was supported by the BMBF (Bundesministerium für Bildung und Forschung) of the Federal Republic of Germany under grant 01M 3061 J. The responsibility for this publication is held by the authors only.

\section{References}

Catedra, M. F.: The CG-FFT Method: application of signal processing techniques to electromagnetics, Artech House, Boston, 1995.

Georgakopoulos, S. V., Birtcher, C. R., and Balanis, C. A.: HIRF Penetration Through Apertures: FDTD Versus Measurements, IEEE Trans. on Electromagnetic Compatibility, 43, 282-294, 2001.

Li, M., Nuebel, J., Drewniak, J. L., Broff, R. E. D., Hubing, T. H., and Doren, T. P. V.: EMI from Airflow Aperture Arrays in Shielding Enclosures- Experiments, FDTD and MoM Modeling, IEEE Trans. on Electromagnetic Compatibility, 42, 265275, 2000.

Olyslager, F., Laermans, E., Zutter, D. D., Criel, S., Smedt, R. D., and Lietaert, N.: Numerical and Experimental Study of the Shielding Effectiveness of a Metallic Enclosure, IEEE Trans. on Antennas and Propagation, 47, 202-213, 1999.

Paoletti, U., Keller, U., Garbe, H., and John, W.: A BiCG-FFT method for shielding effectiveness of PEC enclosures with many apertures, IEE Electronic Letters, 47, 1711-1712, 2003.

Paoletti, U., Garbe, H., and John, W.: Simple Measurement Techniques for the Shielding Effectiveness of Symmetric Enclosures, Book of Abstracts EUROEM 2004, 78, 2004a.

Paoletti, U., Garbe, H., and John, W.: Measurement Technique for the Shielding Effectiveness of Symmetric Enclosures with the Use of the Image Theory, Proc. International Symposium on EMC, Sendai, Japan, 621-624, 2004 b.

Paul, J., Podlozny, V., and Christopoulos, C.: The Use of Digital Filtering Techniques for the Simulation of Fine Features in EMC Problems Solved in the Time Domain, IEEE Trans. on Electromagnetic Compatibility, 45, 238-244, 2003.

Sarkar, T. K., Arvas, E., and Rao, S. M.: Application of FFT and the Conjugate Gradient Method for the Solution of Electromagnetic Radiation from Electrically Large and Small Conducting Bodies, IEEE Trans. on Antennas and Propagation, 34, 635-640, 1986.

Siah, E. S., Sertel, K., Volakis, J., Liepa, V., and Wiese, R.: Coupling Studies and Shielding Techniques for Electromagnetic Penetration Through Apertures on Complex Cavities and Vehicular Platforms, IEEE Trans. on Electromagnetic Compatibility, 45, 245-257, 2003. 